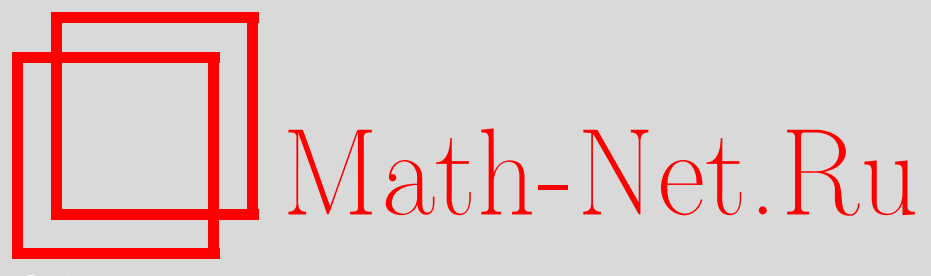

Ю. С. Волков, В. Л. Мирошниченко, О приближении производных скачком интерполяционного сплайна, $\mathrm{Ma}$ тем. заметки, 2011, том 89, выпуск 1, 127-130

DOI: https://doi.org/10.4213/mzm8930

Использование Общероссийского математического портала Math-Net.Ru подразумевает, что вы прочитали и согласны с пользовательским соглашением http://www . mathnet.ru/rus/agreement

Параметры загрузки:

IP : 54.147 .182 .235

26 апреля 2023 г., 13:54:39

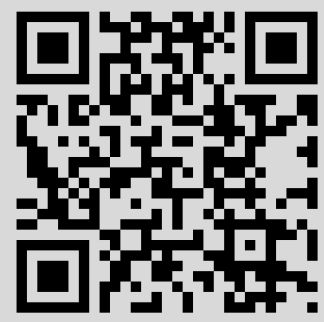




\section{О приближении производных скачком интерполяционного сплайна}

\section{Ю. С. Волков, В. Л. Мирошниченко}

Пусть $\Delta: a=x_{0}<x_{1}<\cdots<x_{N}=b$ - разбиение отрезка $[a, b]$. Тогда $s(x)-$ сплайн степени $2 n-1$ по разбиению $\Delta$, если $s(x) \in C^{2 n-2}[a, b]$, и на каждом подотрезке сетки $\left[x_{i-1}, x_{i}\right], i=1, \ldots, N$, сплайн $s(x)$ есть многочлен степени $2 n-1$. Обозначим

$$
\underline{h}=\min _{1 \leqslant i \leqslant N}\left(x_{i}-x_{i-1}\right), \quad \bar{h}=\max _{1 \leqslant i \leqslant N}\left(x_{i}-x_{i-1}\right)
$$

соответственно наименьший и наибольший шаги разбиений. Считаем, что сплайн $s(x)$ в узлах сетки интерполирует значения некоторой достаточно гладкой функции $f(x)$, т.е. $s\left(x_{i}\right)=f\left(x_{i}\right), i=0, \ldots, N$. Также считаем, что $s(x)$ и $f(x)$ являются $(b-a)$-периодическими, что однозначно определяет сплайн $s(x)$ (см., например, [1], [2]).

Хорошо известно, что полиномиальные сплайны $s(x)$, интерполирующие значения гладкой функции $f(x)$, обладают хорошими аппроксимативными свойствами, и вместе с приближением самой функции $f(x)$ производные сплайна $s^{(k)}(x)$ приближают соответствующие производные $f^{(k)}(x)$ интерполируемой функции (см., например, [1], [2]). И, что самое удивительное, сплайном нечетной степени $2 n-1$ можно приблизить производную $f^{(2 n)}(x)$ интерполируемой функции несмотря на то, что производная любого звена (куска полинома) такого сплайна тождественно равна нулю. Именно величина разрыва

$$
\beta_{i}=s^{(2 n-1)}\left(x_{i}+0\right)-s^{(2 n-1)}\left(x_{i}-0\right)
$$

старшей $(2 n-1)$-й производной сплайна в узле $x_{i}$, отнесенная к величине шага равномерной сетки (назваемая скачком), приближает $(2 n)$-ю производную исходной функции.

По видимому, впервые такой результат был получен в 1967 г. [2], [3]. Альберг, Нильсон, Уолш [2] показали только сам факт приближения, а Куртис, Повелл [3] для кубических сплайнов установили порядок приближения, который оказался достаточно высоким, используя, однако, эвристические аргументы. Для сплайнов пятой степени порядок приближения приведен в работах [4], [5]. Порядок приближения скачком для сплайнов произвольной нечетной степени установлен практически одновременно и независимо в работах [6], [7].

Все указанные результаты о приближении скачком сплайна доказаны для случая равномерной сетки и периодических краевых условий; однако для кубического случая Альберг, Нильсон, Уолш [2] показали, что

$$
\max _{i}\left|\frac{\beta_{i}}{\bar{h}}-f^{(4)}\left(x_{i}\right)\right| \rightarrow 0
$$

на последовательности сеток, стремящихся к равномерным. Кроме того, в работе [8] рассмотрены и иные граничные условия.

Для представления сплайнов и производных удобно использовать базис из нормализованных $B$-сплайнов [1], которые неотрицательны и имеют минимальный носитель из $2 n$ последовательных интервалов разбиения

$$
N_{i}(x)=\left(x_{i+2 n}-x_{i}\right) \cdot(-x)_{+}^{2 n-1}\left[x_{i}, \ldots, x_{i+2 n}\right],
$$

Работа выполнена при поддержке Отделения математических наук РАН (грант № 2009-1.3.8), Интеграционных проектов СО РАН (грант № 2009-81) и проектов СО РАН, выполняемых совместно с УрО РАН (грант № 2009-14). 
причем

$$
\sum_{j=i-2 n+1}^{i} N_{j}(x) \equiv 1, \quad x \in\left[x_{i}, x_{i+1}\right] .
$$

Однако в случае периодических сплайнов для представления более удобны периодические аналоги - периодические $B$-сплайны. Сетку $\Delta$ считаем периодически продолженной за пределы отрезка $[a, b]$; тогда периодические $B$-сплайны определяем так:

$$
\tilde{N}_{i}(x)=\sum_{\nu=-\infty}^{\infty} N_{i+\nu N}(x) .
$$

Отметим, что если $N>2 n$, то в этой сумме при любом значении $x$ не более одного ненулевого слагаемого.

Интерполяционный сплайн $s(x)$ однозначно может быть представлен в виде разложения по базису:

$$
s(x)=\sum_{i=1}^{N} \alpha_{i} \widetilde{N}_{i}(x), \quad x \in[a, b] .
$$

Для разрывов в узлах сетки старшей производной сплайна

$$
\beta_{i}=s^{(2 n-1)}\left(x_{i}+0\right)-s^{(2 n-1)}\left(x_{i}-0\right)
$$

известна [9], [10] система линейных уравнений

$$
\sum_{j=1}^{N} \widetilde{N}_{i}\left(x_{j}\right) \beta_{j}=(2 n-1) !\left\{f\left[x_{i+1}, \ldots, x_{i+2 n}\right]-f\left[x_{i}, \ldots, x_{i+2 n-1}\right]\right\} .
$$

Интересно отметить, что матрицей данной системы является транспонированная матрица от хорошо известной коллокационной матрицы.

Теорема 1. Для равномерной сетки $(\underline{h}=\bar{h}=h)$ имеет место

$$
\frac{\beta_{i}}{h}=f^{(2 n)}\left(x_{i}\right)+o(1) .
$$

ДокАзАТЕЛЬСтво немедленно следует из таких фактов, что по свойствам разделенных разностей

$$
(2 n-1) !\left\{f\left[x_{i+1}, \ldots, x_{i+2 n}\right]-f\left[x_{i}, \ldots, x_{i+2 n-1}\right]\right\}=\frac{x_{i+2 n}-x_{i}}{2 n} f^{(2 n)}\left(\xi_{i}\right)
$$

для некоторого $\xi_{i} \in\left[x_{i}, x_{i+2 n}\right]$, и для равномерной сетки

$$
\sum_{j=1}^{N} \tilde{N}_{i}\left(x_{j}\right)=\sum_{j=1}^{N} \tilde{N}_{j}\left(x_{i}\right)=1
$$

теорема 1 была доказана в [2]. Там же в кубическом случае была доказана подобная теорема для последовательности сеток, стремящихся к равномерным. Теперь мы можем сформулировать и в общем случае такое утверждение.

Теорема 2. Пусть последовательность сеток $\{\Delta\}$ такова, что $\bar{h} / \underline{h} \rightarrow 1 ;$ тогда

$$
\frac{\beta_{i}}{\bar{h}} \rightarrow f^{(2 n)}\left(x_{i}\right)
$$


Если же последовательность сеток не стремится к равномерной, т.е. не выполнено условие $\bar{h} / \underline{h} \rightarrow 1$, то в общем случае неравномерной сетки не ясно, что понимать под скачком: на какой из шагов сетки надо делить величину $\beta_{i}$ ? Можно попробовать под скачком понимать величину $\beta_{i} / H_{i}$, где, например, $H_{i}$ - полусумма шагов слева и справа или какое-нибудь более сложное выражение.

Перепишем систему (1) в виде

$$
\sum_{j=1}^{N} \frac{2 n}{x_{i+2 n}-x_{i}} \widetilde{N}_{i}\left(x_{j}\right) \beta_{j}=(2 n) ! f\left[x_{i}, \ldots, x_{i+2 n}\right] .
$$

Очевидно, что $\beta_{i} / H_{i}$ будет приближать величину $f^{(2 n)}\left(x_{i}\right)$ только в том случае, если будет выполнено условие

$$
\sum_{j=1}^{N} \frac{2 n}{x_{i+2 n}-x_{i}} H_{j} \widetilde{N}_{i}\left(x_{j}\right)=1 .
$$

Несмотря на то, что в сумме слева в (2) только лишь $2 n-1$ слагаемых отличны от нуля (при $N>2 n$ ), не ясно какое предложить выражение для $H_{i}$, чтобы равенства (2) выполнялись для всех $i$.

Тем не менее, выражение для $H_{i}$ для некоторых специальных сеток можно подобрать.

Теорема 3. Если расположение узлов сетки таково, что

$$
x_{2 k+1}-x_{2 k}=h_{0}, \quad x_{2 k}-x_{2 k-1}=h_{1} \quad \text { для всех } \quad k,
$$

то равенства (2) выполняются для всех $і$ при $H_{i}=\left(x_{i+1}-x_{i-1}\right) / 2$ u, следовательно,

$$
\frac{\beta_{i}}{H_{i}}=f^{(2 n)}\left(x_{i}\right)+o(1) \text {. }
$$

ДокАзАтельство. Такое разбиение отрезка $[a, b]$ хотя и является неравномерным, но имеется лишь 2 различных шага сетки $h_{0}$ и $h_{1}$, которые строго чередуются. На такой сетке будут всего 2 принципиально различных $B$-сплайна, остальные получаются их сдвигом. Причем поскольку носитель всегда состоит из четного числа интервалов сетки, то эти различные $B$-сплайны получаются один из другого симметричным отражением относительно середины носителя. Следовательно, $N_{i}\left(x_{i+k}\right)=N_{i+1}\left(x_{i+2 n+1-k}\right)$. В этом случае

$$
\begin{aligned}
\sum_{j=1}^{N} \widetilde{N}_{i}\left(x_{j}\right) & =N_{i}\left(x_{i+1}\right)+\cdots+N_{i}\left(x_{i+2 n-1}\right)=\sum_{j=0}^{n-1} N_{i}\left(x_{i+2 j+1}\right)+\sum_{j=1}^{n-1} N_{i}\left(x_{i+2 j}\right) \\
& =\sum_{j=0}^{n-1} N_{i-2 j}\left(x_{i+1}\right)+\sum_{j=1}^{n-1} N_{i+1}\left(x_{i+2 n+1-2 j}\right) \\
& =\sum_{j=0}^{n-1} N_{i-2 j}\left(x_{i+1}\right)+\sum_{j=1}^{n-1} N_{i+1-2 n+2 j}\left(x_{i+1}\right)=N_{i-2 n+2}\left(x_{i+1}\right)+\cdots+N_{i}\left(x_{i+1}\right)=1 .
\end{aligned}
$$

Тогда, учитывая, что

$$
\frac{2 n}{x_{i+2 n}-x_{i}}=\frac{2}{h_{0}+h_{1}}, \quad H_{j}=\frac{h_{0}+h_{1}}{2},
$$

получаем выполнение равенств (2) для всех $i$. Теорема доказана. 


\section{СПИСОК ЦИТИРОВАННОЙ ЛИТЕРАТУРЫ}

[1] Ю. С. Завьялов, Б. И. Квасов, В. Л. Мирошниченко, Методы сплайн-функиий, Наука, M., 1980. [2] J. H. Ahlberg, E. N. Nilson, J. L. Walsh, The Theory of Splines and their Applications, Academic Press, New York, 1967. [3] A. R. Curtis, M. J. D. Powell, Using Cubic Splines to Approximate Functions of One Variable to Prescribed Accuracy, Atomic Energy Research Establishment, Report R5602, Harwell, 1967. [4] D. J. Fyfe, J. Inst. Math. Appl., 7:3 (1971), 398-406. [5] Б. С. Киндалев, Вычисл. системы, 87, ИМ СО АН СССР, Новосибирск, 1981, 18-24. [6] Б. С. Киндалев, Вычисл. системы, 93, ИМ СО АН СССР, Новосибирск, 1982, 39-52. [7] T. R. Lucas, SIAM J. Numer. Anal., 19:5 (1982), 1051-1066. [8] T. R. Lucas, SIAM J. Numer. Anal., 11:3 (1974), 569-584. [9] Ю. С. Волков, Равномерная сходимость производных интерполяиионных сплайнов нечетной степени, Препринт № 62, ИМ СО АН СССР, Новосибирск, 1984. [10] Ю. С. Волков, Сиб. журн. вычисл. матем., 13:3 (2010), 243-253.

Ю. С. Волков

Институт математики им. С. Л. Соболева СО РАН, Новосибирский государственный университет, г. Новосибирск E-mail: volkov@math.nsc.ru

\section{В. Л. Мирошниченко}

Институт математики им. С. Л. Соболева СО РАН, Новосибирский государственный университет, г. Новосибирск E-mail: miroshn@math.nsc.ru
Поступило

19.04.2010 